\title{
The Casimir effect for a cavity in the spacetime with an extra dimension
}

\author{
Cheng Hong-Bo* \\ Department of Physics, East China University of Science and Technology, \\ Shanghai 200237, China
}

\begin{abstract}
We reexamine the Casimir effect for the rectangular cavity with two or three equal edges in the presence of compactified universal extra dimension. We derive the expressions for the Casimir energy and discuss the nature of Casimir force. We show analytically the extra-dimension corrections to the standard Casimir effect to put forward a new method of exploring the existence of extra dimensions of the Universe.
\end{abstract}

PACS number(s): 11.10.Kk, 04.62.+v

*E-mail address: hbcheng@public4.sta.net.cn 
Kaluza and Klein presented the thought that our spacetime has more than three spatial dimensions over decades ago in order to unify gravity and classical electrodynamics $[1,2]$. Now the idea of extra dimensions attracts more and more interests of the physical community. It is fundamental for string theory to invoke seven additional spatial dimensions to unify the quantum mechanics and gravity. Whether the order of the compactification scale of the extra dimensions is much larger or smaller remains unexplained. Some models of string theory predicted that the typical size of universal extra dimensions (UXDs) is about $10^{-35} \mathrm{~m}$ which is beyond our recent experimental reach $[3$, 4]. Recently the Kaluza-Klein idea is considered in the context of the brane paradigm, which could lead to a solution to the hierarchy problem [5-7]. The brane world scenario $[8,9]$ stipulates that the non-gravitational physics like Standard Model is confined to a four-dimensional Universe called brane that is embedded in a $(4+n)$-dimensional spacetime with $n$ compactified extra dimensions. Only gravitational field is allowed to propagate through the whole spacetime while the possibility that the additional dimensions may be much larger than we thought in the past was showed.

The Casimir effect is essentially a direct consequence of quantum field theory because of a change in the spectrum of vacuum oscillations when the quantization volume is bounded, or some background field is presented. The effect becomes one of the most interesting macroscopic manifestations of the nontrivial properties of the physical vacuum. The Casimir effect has been a subject of extensive research [10-16]. Nowadays the Casimir effect is used as a powerful method for the research on new physics beyond the standard model. We apply the tool to a lot of topics such as the right order of the cosmological constant, extra dimensions, etc..

It is necessary to explore the dimensionality and structure of our Universe in various directions. If our world has six or seven additional spatial dimensions, the new phenomena related to the existence of these dimensions should be observed. A lot of progresses of studying the UXDs by means of Casimir effect were made [17, 18]. The authors of [18] compared the expression of Casimir force for parallel plates in the presence of one extra dimension with the experimental results and the approximate values of the compactification scale of extra dimension were obtained.

In this paper, we investigate the extra-dimension correction to the Casimir effect for a cavity in detail to put forward a new method to explore the existence of extra dimensions of our Universe. There will be influence from UXD on the nature of Casimir force between the configuration boundaries that confine the field in the spacetime with extra dimensions. It was shown that the sign of Casimir energy for a rectangular cavity with two or three equal edges in four-dimensional Minkowski spacetime is positive or negative respectively for the case of massless scalar field [14, 19]. Here we also discuss the Casimir effect of this kind of field satisfying Dirichlet boundary conditions within a rectangular with two or three equal edges in the spacetime with one extra dimension for the sake of comparing results of $[14,19]$ and recent ours. Having derived the total energy, we regularize the energy by means of Epstein zeta function to obtain the Casimir energy. We find that the Casimir force is always repulsive if the lengths of edges are sufficiently large with respect to the size of UXD. 
In order to derive and calculate the Casimir energy, in the Kaluza-Klein theory we chose the Lagrangian density of a simple model of scalar field in $(4+n)$-dimensional spacetime like,

$$
\mathcal{L}=\frac{1}{2} \partial_{A} \Phi \partial^{A} \Phi
$$

with the metric $(+----)$. Here the field $\Phi\left(x^{A}\right)=\Phi\left(x^{\mu}, y\right), A=0,1,2,3,5$ is the function of four-dimensional coordinates $x^{\mu}$ with $\mu=0,1,2,3$ as well as an extra coordinate $y$. In the five-dimensional spacetime like $M^{4} \times S^{1}$, with the compactification of extra dimension on a circle $S^{1}$ of radius $L$, the field $\Phi\left(x^{A}\right)$ can be expanded in the harmonics as follow,

$$
\Phi\left(x^{\mu}, y\right)=\sum_{n=-\infty}^{\infty} \phi_{n}\left(x^{\mu}\right) e^{\frac{i n y}{L}}
$$

By substituting the expansion (2) into (1), the Lagrangian density (1) becomes,

$$
\mathcal{L}=\frac{1}{2} \partial_{\mu} \phi_{0} \partial^{\mu} \phi_{0}+\sum_{k=1}^{\infty}\left(\partial_{\mu} \phi_{k} \partial^{\mu} \phi_{k}^{*}+\frac{k^{2}}{L^{2}} \phi_{k} \phi_{k}^{*}\right)
$$

where $L$ is the radius of UXD as mentioned above. The scalar fields $\phi_{k}$ satisfy the free Klein-Gordon equations,

$$
\left(\partial_{\mu} \partial^{\mu}-\frac{k^{2}}{L^{2}}\right) \phi_{k}(x)=0
$$

here $k=0,1,2, \cdots$. The fields are confined in the interior of rectangular cavity $\Omega$ with $p$ edges. We consider the case of Dirichlet boundary conditions, i.e., $\left.\phi_{k}(x)\right|_{\partial \Omega}=0$. The total energy density of the fields in the interior of $\Omega$ is thus given by,

$$
\varepsilon_{p}=\int d^{3-p} k \sum_{\{n\}=1}^{\infty} \sum_{n=0}^{\infty} \frac{1}{2} \sqrt{k_{T}^{2}+\left(\frac{n_{1} \pi}{R_{1}}\right)^{2}+\left(\frac{n_{2} \pi}{R_{2}}\right)^{2}+\cdots+\left(\frac{n_{p} \pi}{R_{p}}\right)^{2}+\frac{n^{2}}{L^{2}}}
$$

Following [11, 14], Eq. (5) becomes,

$$
\begin{aligned}
\varepsilon_{p}=-\frac{1}{2^{4-p}} \Gamma( & \left.-\frac{4-p}{2}\right) E_{p+1}\left(\frac{\pi^{2}}{R_{1}^{2}}, \frac{\pi^{2}}{R_{2}^{2}}, \cdots, \frac{\pi^{2}}{R_{p}^{2}}, \frac{1}{L^{2}} ;-\frac{4-p}{2}\right) \\
- & \frac{1}{2} \Gamma\left(-\frac{4-p}{2}\right) E_{p}\left(\frac{\pi^{2}}{R_{1}^{2}}, \frac{\pi^{2}}{R_{2}^{2}}, \cdots, \frac{\pi^{2}}{R_{p}^{2}} ;-\frac{4-p}{2}\right)
\end{aligned}
$$

where Epstein zeta function $E_{p}\left(a_{1}, a_{2}, \cdots, a_{p} ; s\right)$ is defined as,

$$
E_{p}\left(a_{1}, a_{2}, \cdots, a_{p} ; s\right)=\sum_{\{n\}=1}^{\infty}\left(\sum_{j=1}^{p} a_{j} n_{j}^{2}\right)^{-s}
$$

$\{n\}$ stands for a short notation of $n_{1}, n_{2}, \cdots, n_{p}, n_{a}$ a positive integer.

In addition, the Casimir force can be denoted as,

$$
f_{C}=-\frac{\partial \varepsilon_{p}}{\partial L_{p}}
$$


We consider $p=2$ case in which the fields are confined in the interior of cavity with two equal edges such as $R_{1}=R_{2}=R$ in the presence of one compactified universal extra dimension. For the case of four-dimensional Minkowski spacetime, the Casimir energy of two-edge hypercube keeps positive $[14,19]$. In the four-dimensional spacetime with one extra dimension, the expression (6) is reduced to,

$$
\varepsilon_{2}=-\frac{1}{4} \Gamma(-1) E_{3}\left(\frac{\pi^{2}}{R^{2}}, \frac{\pi^{2}}{R^{2}}, \frac{1}{L^{2}} ;-1\right)-\frac{1}{4} \Gamma(-1) E_{2}\left(\frac{\pi^{2}}{R^{2}}, \frac{\pi^{2}}{R^{2}} ;-1\right)
$$

By regularizing Eq.(9), we obtain the Casimir energy,

$$
\begin{aligned}
\varepsilon_{2}= & -\frac{1}{8 \pi^{\frac{5}{2}}} \Gamma\left(\frac{3}{2}\right) \zeta(3) \frac{1}{L^{2}}+\frac{1}{4 \pi^{4}} \mu \Gamma(2) \zeta(4) \frac{1}{L^{2}}+\frac{1}{2 \pi^{\frac{1}{2}} \mu^{\frac{1}{2}}} \frac{1}{L^{2}} \sum_{n_{2}, n=1}^{\infty}\left(\frac{n}{n_{2}}\right)^{\frac{3}{2}} K_{\frac{3}{2}}\left(2 \mu n_{2} n\right) \\
& -\frac{1}{8 \pi^{\frac{11}{2}}} \mu^{2} \Gamma\left(\frac{5}{2}\right) \zeta(5) \frac{1}{L^{2}}-\frac{1}{2 \pi} \frac{1}{L^{2}} \sum_{n_{2}, n=1}^{\infty}\left(\frac{n}{n_{2}}\right)^{2} K_{2}\left(2 \mu n_{2} n\right) \\
& -\frac{1}{2 \mu^{2}} \frac{1}{L^{2}} \sum_{k=0}^{\infty} \frac{16^{-k}}{k !} \prod_{j=1}^{k}\left[9-(2 j-1)^{2}\right] \\
& \times \sum_{n_{1}, n_{2}, n=1}^{\infty} n_{1}^{-k-2}\left(\pi^{2} n_{2}^{2}+\mu^{2} n^{2}\right)^{-\frac{k-1}{2}} \exp \left[-2 n_{1}\left(\pi^{2} n_{2}^{2}+\mu^{2} n^{2}\right)^{\frac{1}{2}}\right] \\
& +\frac{1}{2 \pi^{\frac{1}{2}} \mu^{2}} \Gamma\left(\frac{3}{2}\right) \zeta(3) \frac{1}{L^{2}}-\frac{1}{2 \pi \mu^{2}} \frac{1}{L^{2}} \Gamma(2) \zeta(2) \beta(2)
\end{aligned}
$$

where

$$
\mu=\frac{R}{L}
$$

and

$$
\beta(s)=\sum_{n=0}^{\infty} \frac{(-1)^{n}}{(2 n+1)^{s}}
$$

If the lengths of cavity are large enough like $\mu>>1$, the Casimir energy density (10) becomes,

$$
\varepsilon_{2}(\mu>>1)=-\frac{1}{8 \pi^{\frac{11}{2}}} \mu^{2} \Gamma\left(\frac{5}{2}\right) \zeta(5) \frac{1}{L^{2}}
$$

In the case of $\mu<<1$, then the energy density can be denoted as,

$$
\begin{aligned}
\varepsilon_{2}(\mu<<1)= & -\frac{1}{2 \mu^{2}} \frac{1}{L^{2}} \sum_{k=0}^{\infty} \frac{16^{-k}}{k !} \prod_{j=1}^{k}\left[9-(2 j-1)^{2}\right] \\
& \times \sum_{n_{1}, n_{2}, n=1}^{\infty} n_{1}^{-k-2}\left(\pi^{2} n_{2}^{2}+\mu^{2} n^{2}\right)^{-\frac{k-1}{2}} \exp \left[-2 n_{1}\left(\pi^{2} n_{2}^{2}+\mu^{2} n^{2}\right)^{\frac{1}{2}}\right] \\
& +\frac{1}{2 \pi^{\frac{1}{2}} \mu^{2}} \Gamma\left(\frac{3}{2}\right) \zeta(3) \frac{1}{L^{2}}-\frac{1}{2 \pi \mu^{2}} \frac{1}{L^{2}} \Gamma(2) \zeta(2) \beta(2) \\
> & 0
\end{aligned}
$$


We show the curves of the Casimir energy density for a rectangular cavity with two equal edges in the spacetime with one UXD in Figure 1. Furthermore, numerical calculation show that there exists a particular critical ratio $\mu_{C}=5.526$ such that the energy density $\varepsilon_{2}<0$ if $\frac{R}{L}>\mu_{C}$ while in the opposite case $\frac{R}{L}<\mu_{C}$, the energy density remains positive. At $\frac{R}{L}>\mu_{C}$, the nature of Casimir force is repulsive.

In this section we consider the Casimir effect for a cube with equal edges like $R_{1}=R_{2}=R_{3}=R$ in the spacetime with one UXD. The total energy density for the system can be derived as,

$$
\varepsilon_{3}=\frac{1}{2} E_{4}\left(\frac{\pi^{2}}{R^{2}}, \frac{\pi^{2}}{R^{2}}, \frac{\pi^{2}}{R^{2}}, \frac{1}{L^{2}} ;-\frac{1}{2}\right)+\frac{1}{2} E_{3}\left(\frac{\pi^{2}}{R^{2}}, \frac{\pi^{2}}{R^{2}}, \frac{\pi^{2}}{R^{2}} ;-\frac{1}{2}\right)
$$

By regularizing the expression (15), we obtain the Casimir energy density,

$$
\begin{aligned}
& \varepsilon_{3}=\frac{1}{182} \frac{1}{L}-\frac{3}{32 \pi^{\frac{7}{2}}} \mu \Gamma\left(\frac{3}{2}\right) \zeta(3) \frac{1}{L}-\frac{1}{8 \pi} \frac{1}{L} \sum_{n_{3}, n=1}^{\infty} \frac{n}{n_{3}} K_{1}\left(2 \mu n_{3} n\right) \\
& +\frac{3}{32 \pi^{5}} \mu^{2} \Gamma(2) \zeta(4) \frac{1}{L}+\frac{\mu^{\frac{1}{2}}}{4 \pi^{\frac{3}{2}}} \frac{1}{L} \sum_{n_{3}, n=1}^{\infty}\left(\frac{n}{n_{3}}\right)^{\frac{3}{2}} K_{\frac{3}{2}}\left(2 \mu n_{3} n\right) \\
& +\frac{1}{8 \pi^{\frac{1}{2}}} \frac{1}{\mu} \frac{1}{L} \sum_{k=0}^{\infty} \frac{16^{-k}}{k !} \prod_{j=1}^{k}\left[4-(2 j-1)^{2}\right] \\
& \times \sum_{n_{2}, n_{3}, n=1}^{\infty} n_{2}^{-k-\frac{3}{2}}\left(\pi^{2} n_{3}^{2}+\mu^{2} n^{2}\right)^{-\frac{2 k-1}{4}} \exp \left[-2 n_{2}\left(\pi^{2} n_{3}^{2}+\mu^{2} n^{2}\right)^{\frac{1}{2}}\right] \\
& -\frac{1}{32 \pi^{\frac{13}{2}}} \mu^{3} \Gamma\left(\frac{5}{2}\right) \zeta(5) \frac{1}{L}-\frac{\mu}{8 \pi^{2}} \frac{1}{L} \sum_{n_{3}, n=1}^{\infty}\left(\frac{n}{n_{3}}\right)^{2} K_{2}\left(2 \mu n_{3} n\right) \\
& -\frac{1}{8 \pi \mu} \frac{1}{L} \sum_{k=0}^{\infty} \frac{16^{-k}}{k !} \prod_{j=1}^{k}\left[9-(2 j-1)^{2}\right] \\
& \times \sum_{n_{2}, n_{3}, n=1}^{\infty} n_{2}^{-k-2}\left(\pi^{2} n_{3}^{2}+\mu^{2} n^{2}\right)^{-\frac{k-1}{2}} \exp \left[-2 n_{2}\left(\pi^{2} n_{3}^{2}+\mu^{2} n^{2}\right)^{\frac{1}{2}}\right] \\
& -\frac{1}{4 \pi^{\frac{1}{2}}} \frac{1}{\mu} \frac{1}{L} \sum_{k=0}^{\infty} \frac{16^{-k}}{k !} \prod_{j=1}^{k}\left[4-(2 j-1)^{2}\right] \\
& \times \sum_{n_{1}, n_{2}, n_{3}, n=1}^{\infty} n_{1}^{-k-\frac{3}{2}}\left(\pi^{2} n_{2}^{2}+\pi^{2} n_{3}^{2}+\mu^{2} n^{2}\right)^{-\frac{2 k-1}{4}} \\
& \times \exp \left[-2 n_{1}\left(\pi^{2} n_{2}^{2}+\pi^{2} n_{3}^{2}+\mu^{2} n^{2}\right)^{\frac{1}{2}}\right] \\
& -\frac{\pi}{96} \frac{1}{\pi} \frac{1}{L}+\frac{3}{16 \pi^{\frac{3}{2}}} \frac{1}{\mu} \Gamma\left(\frac{3}{2}\right) \zeta(3) \frac{1}{L} \\
& +\frac{\pi^{\frac{1}{2}}}{2} \frac{1}{\mu} \frac{1}{L} \sum_{n_{2}, n_{3}=1}^{\infty} \frac{n_{3}}{n_{2}} K_{1}\left(2 \pi n_{2} n_{3}\right)-\frac{1}{4 \pi^{2}} \frac{1}{\mu^{2}} \Gamma(2) \zeta(2) \beta(2) \frac{1}{L} \\
& -\frac{1}{2 \mu} \frac{1}{L} \sum_{k=0}^{\infty} \frac{(16 \pi)^{-k}}{k !} \prod_{j=1}^{k}\left[4-(2 j-1)^{2}\right] \\
& \times \sum_{n_{1}, n_{2}, n_{3}=1}^{\infty} n_{1}^{-k-\frac{3}{2}}\left(n_{2}^{2}+n_{3}^{2}\right)^{-\frac{2 k-1}{4}} \exp \left[-2 \pi n_{1}\left(n_{2}^{2}+n_{3}^{2}\right)^{\frac{1}{2}}\right]
\end{aligned}
$$


The Casimir energy density in unit of $\frac{1}{L}$ for an equal-edged cube in the presence of one UXD is depicted in Figure 2. The sign of the energy density keeps negative, in accordance with the results in $[14,19]$. The derivation and numerical calculation according to (16) show that there also exists a special ratio $\mu_{f}=1.2$. We find that the Casimir force is attractive if $\frac{R}{L}<\mu_{f}$ and it is repulsive at $\frac{R}{L}>\mu_{f}$. Therefore, the Casimir force may be repulsive for the rectangular cavity with three equal edges in the Universe with one UXD, in contrast with the same problem in the four-dimensional Minkowski spacetime. The nature of Casimir force depends on the compactified universal extra dimension.

The Casimir effect for a cavity in the presence of compactified universal extra dimensions is different to that in the four-dimensional spacetime. This means that we give out a new method for exploring the existence of extra dimensions of our Universe. Here we have discussed the Casimir effect for a rectangular cavity with two or three equal edges in the world with one extra dimension. We derive and calculate the Casimir energy. We also discuss the nature of Casimir force between boundaries. We show that there exist influence of extra dimensions on the Casimir effect. For a $p=2$ equal-edge cavity, the sign of Casimir energy will become negative if the edges are sufficiently large, meaning that the edges are larger enough than the radius of UXD or the energy will be positive. The boundaries repulse each other in the case of negative Casimir energy. For the $p=3$ equal-edge cavity, the Casimir energy keeps negative. The Casimir force between boundaries is attractive if we choose $\frac{R}{L}<1.2$ and it is repulsive at $\frac{R}{L}>1.2$, which is different from the results in $[14,19]$. The influence from UXD on the Casimir effect is manifest.

This work is supported by the Basic Theory Research Fund of East China University of Science and Technology, grant No. YK0127312 and partly supported by the Shanghai Municipal Science and Technology Commission No.04dz05905. 


\section{References}

[1] Kaluza T 1921 Sitz. Preuss. Akad. Wiss. Phys. Math. K1 966

[2] Klein O 1926 Z. Phys. 37895

[3] Green M B, Schwarz J H, Witten E 1987 Superstring Theory (Cambridge Univ. Press)

[4] Horova P, Witten E 1996 Nucl. Phys. B460 506

[5] Arkani-Hamed N, Dimopoulos S, Dvali G 1998 Phys. Lett. B429 263

[6] Antoniadis I 1990 Phys. Lett. B246 317

Antoniadis I, Arkani-Hamed N, Dimopoulos S, Dvali G 1998 Phys. Lett. B436 257

[7] Arkani-Hamed N, Dimopoulos S, Dvali G 1999 Phys. Rev. D59 086004

[8] Randall L, Sundrum R 1999 Phys. Rev. Lett. 834690

[9] Randall L, Sundrum R 1999 Phys. Rev. Lett. 833370

[10] Casimir H B G 1948 Proc. Nederl. Akad. Wetenschap 51793

[11] Plunien G, Muller B, Greiner W 1986 Phys. Rep. 13487

Elizalde E, Odintsov S D, Romeo A, Bytsenko A A, Zerbini S 1994 Zeta Regularization Techniques with Applications (World Scientific Publishing Co. Pte. Ltd.)

Elizalde E 1995 Ten Physical Applications of Spectral Zeta Fnctions (Springer-Verlag)

[12] Bordag M, Mohideen, Mostepanenko V M 2001 Phys. Rep. 3531

Milton K A 2001 The Casimir Effect, Physical manifestations of zero-point energy (World Scientific Publishing Co. Pte. Ltd.)

Bordag M, Elizalde E, Kirsten K 1996 J. Math. Phys. 37895

Bordag M, Elizalde E, Kirsten K, Leseduarte 1997 Phys. Rev. D56 4896

Elizalde E, Bordag M, Kirsten K 1998 J. Phys. A31 1743

[13] Maclay J G 2000 Phys. Rev. A61 052110

[14] Li X, Cheng Hongbo, Li J, Zhai X 1997 Phys. Rev. D56 2155

[15] Cheng Hongbo, Li X 2001 Chin. Phys. Lett. 181163

[16] Cheng Hongbo 2002 J. Phys. A: Math. Gen. 352205

Elizalde E, Nojiri S, Odintsov S D, Ogushi S 2003 Phys. Rev. D67 063515

Cognola G, Elizalde E, Nojiri S, Odintsov S D, Zerbini S 2004 Mod. Phys. Lett. A19 1435

[17] Appelquist T, Cheng H C, Dobrescu B A 2001 Phys. Rev. D64 035002 
[18] Poppenhaeger K, Hossenfelder S, Hofmann S, Bleicher M 2004 Phys. Lett. B582 1 Saharian A A, Setare M R 2003 Phys. Lett. B552 119

[19] Caruso F, Neto N P, Svaiter B F, Svaiter N F 1991 Phys. Rev. D43 1300 
Figure 1: The curve of Casimir energy density as function of $R / L$ for $p=2$ equal-edge rectangular cavity in the spacetime with one UXD.

Figure 2: The curve of Casimir energy density as function of $R / L$ for $p=3$ equal-edge rectangular cavity in the spacetime with one UXD. 